\title{
Tight Binding Simulation of Quantum Transport in Interband Tunneling Devices
}

\author{
MATSUTO OGAWA*, RYUICHIRO TOMINAGA and TANROKU MIYOSHI \\ Department of Electrical and Electronics Engineering, Kobe University, \\ 1 Rokkodai, Nada, Kobe, 657-8501, Japan
}

\begin{abstract}
We have studied quantum transport in both $\mathrm{Si}$ and GaAs interband tunneling diodes (ITD's). In the simulation, a non-equilibrium Green's function method based on an empirical tight binding theory has been used to take into account evanescent-wave matching at interfaces and realistic band structures. Comparison has been made between the results of our multiband (MB) model and those of conventional two-band (2B) model. As a result, it is found that the current-voltage $(I-V)$ characteristics of the Si ITD have considerably smaller peak current density than the conventional 2B model, since our MB model reflects correctly the indirect gap band structure. On the other hand, in the GaAs ITD, there is small difference between the two models, because tunneling occurs between the conduction band and the valence band at $\Gamma$ point. It is also found that the matching of evanescent electron modes is essentially necessary to include the valley-mixing effects at the tunneling interfaces.
\end{abstract}

Keywords: Interband tunneling diode; Non-equilibrium Green's function; Tight-binding approximation; Complex band structure; Evanescent electron waves

\section{INTRODUCTION}

Interband tunneling diodes (ITD's) or Esaki diodes have revived and attracted much attention again $[1,2,3]$, since they are very suitable for device miniaturization below $0.1 \mu \mathrm{m}$, whereas, in such scale, conventional FETs suffer with the short channel effects and do not operate properly. The functionality of ITD's such as negative differential conductance (NDC) is expected to improve device performance as well as reduce device numbers in $\mathrm{Si}$ ULSIs and/or GaAs ICs. Although room temperature operations have been achieved, thorough understanding of the device physics is still necessary to optimize the device performance such as high peak-to-valley $(\mathrm{P} / \mathrm{V})$ ratio. To date, only simple two-band model has been applied to analyze the characteristics of such ITD's, but failed to reflect complex band structures of the materials. In order to simulate quantum transport in such devices realistically, we have to include properly the full band structure effect such as the band mixing between the conduction band and the valence band as well as nonparabolicity.

\footnotetext{
*Corresponding author. Fax: +81-78-803-6074, e-mail: ogawa@eedept.kobe-u.ac.jp
} 
In this paper, we present calculations of quantum electron transport in Si and GaAs ITD's based on a non-equilibrium Green's function formalism. In the next section, we describe a tightbinding model and the Green's function method. In our procedure, we employ an empirical $s p^{3} s^{*}$ tight-binding (TB) model and an evanescent-wavematching method to include the valley mixing and nonparabolicity effects, in addition, possible existence of evanescent modes at heterointerfaces $[4,5]$. We show numerical results compared with those calculated by the conventional two band model for a GaAs ITD and a Si ITD, respectively. Finally, we summarize our conclusion.

\section{THEORY}

\section{Tight-binding Model}

We treat carrier transport in the $\left[\begin{array}{lll}0 & 0 & 1\end{array}\right]$ direction in quantum structures made of zinc-blende (ZB) or diamond crystals. We use an empirical tightbinding model [6] with a basis of five orbitals per atom $\left(s, p_{x}, p_{y}, p_{z}, s^{*}\right)$ to expand the electronic wave functions. Then the total electron wave function for a ZB crystal can be expressed in terms of the Bloch sum of the anion $(a)$ and cation (c) states as

$$
|\Psi(z)\rangle=\sum_{L, \alpha^{a}, \alpha^{c}, \boldsymbol{k}_{\|}}\left[C_{L, \alpha^{a}, \boldsymbol{k}_{\|}}\left|\boldsymbol{k}_{\|}, L, \alpha^{a}\right\rangle+C_{L, \alpha^{c}, \boldsymbol{k}_{\|}}\left|\boldsymbol{k}_{\|}, L, \alpha^{c}\right\rangle\right],
$$

where $\left|\boldsymbol{k}_{\|}, L, \alpha^{b}\right\rangle$ denotes a Bloch sum of $\alpha^{b}$-like $(b=a, c)$ atomic orbitals associated with the inplane wave vector $\boldsymbol{k}_{\|}, L$ labels the index of the layer comprised of both the anion and cation atoms. (For a diamond lattice, we need only to set the anion and cation atoms are chemically identical.)

The Schrödinger equation for the $\mathrm{ZB}$ crystal is expressed as

$$
(H-E)|\Psi(z)\rangle=0
$$

where $H$ is the Hamiltonian and $E$ is the energy. Projecting the Schrödinger equation on atomic orbitals located at atomic layer $l$ and assuming nearest neighbor coupling, we obtain equations relating the coefficients at layer $l$ to those at layers $l \pm 1$. In the ZB bulk crystal, since anions and cations are located periodically in $z$ direction, we can finally obtain the following eigenvalue equation

$$
T_{c} T_{a}\left(\begin{array}{l}
\boldsymbol{C}_{a} \\
\boldsymbol{C}_{c}
\end{array}\right)=\exp \left(-i k_{z} \Delta\right)\left(\begin{array}{l}
\boldsymbol{C}_{a} \\
\boldsymbol{C}_{c}
\end{array}\right)
$$

where $T_{a}$ and $T_{c}$ are transfer matrices of each layer, defined as

$$
T_{b}=\left(\begin{array}{cc}
-H_{k, l-1}^{(b)-1} H_{l, l}^{(b)} & -H_{l, l-1}^{(b)-1} H_{l, l+1}^{(b)} \\
\mathbf{1} & \mathbf{0}
\end{array}\right) .
$$

Here $H_{l, l-1}^{(b)}$ etc., denote the matrix elements of the Hamiltonian projected onto the atomic orbitals and $C_{b}(b=c$ or $a)$ is comprised of five elements.

The $E-k_{z}$ energy-band diagram of the crystal is obtained by solving the eigenvalue Eq. (3). It should be noted that the eigenvalue equation gives both real and complex solutions of $k_{z}$ (a set of 10 wave numbers in all) $[4,5]$. The real $k_{z}$ produces the Bloch states and the complex $k_{z}$ produces evanescent states which grow $\left(\operatorname{Re} k_{z}>0\right)$ or decay $\left(\boldsymbol{R e} k_{z}<0\right)$ in $z$ direction, where $\mathbf{R e}$ implies the real part of the complex quantity.

At a tunneling junction of an ITD, where the translational symmetry in $z$ direction is lacking, these evanescent modes must be taken into account to match the impinging electron waves to those propagating into the other side. For this purpose, we reorder the incident bulk states so that the first 5 states $(j=1, \ldots, 5)$ correspond to states which propagate or decay in $+z$ direction, while last 5 states $(j=6, \ldots, 10)$ correspond to those propagating or decaying in $-z$ direction, that is, the real parts of the wave vector for the first 5 states are positive $\left(\boldsymbol{R e} k_{z}^{j}>0\right)$, whereas they are negative for the last $5\left(\operatorname{Re} k_{z}^{j}<0\right)$. 
To relate the coefficients of the $L$-th layer to those of the $(L+1)$-th layer using the $+z$ propagating modes $\left|k_{z}^{j}\right\rangle(j=1, \ldots, 5)$, we need only to calculate the following equation:

$$
\left[\begin{array}{c}
C_{l_{(L+1)}} \\
C_{l_{(L+1)}+1}
\end{array}\right]=X \cdot K_{z} \cdot X^{-1}\left[\begin{array}{c}
C_{l_{L}} \\
C_{l_{L}+1}
\end{array}\right]
$$

where

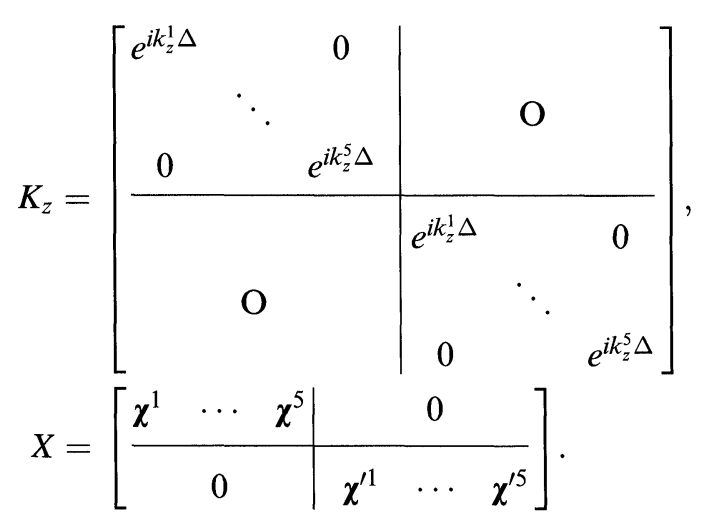

In the above equations, $k_{z}^{j}$ is the $j$-th propagating wave number and $\chi^{j}$ and $\chi^{\prime j}$ are corresponding eigenvectors, respectively. At the tunneling interface, the matching method duly includes the matching condition of the evanescent modes as well as the real modes.

\section{Non-equilibrium Green's Functions}

Two non-equilibrium Green's functions, $G^{<}$and $G^{R}$, are used for the analysis of quantum transport in the device. The Green's functions in the MB space are defined as

$$
\begin{aligned}
& G_{\alpha, L, \alpha^{\prime}, L^{\prime}}^{<}\left(\boldsymbol{k}_{\|} ; t, t^{\prime}\right)=\frac{i}{\hbar}\left\langle c_{\alpha^{\prime}, L^{\prime}, \boldsymbol{k}_{\|}}^{\dagger}\left(t^{\prime}\right) c_{\alpha, L, \boldsymbol{k}_{\|}}(t)\right\rangle, \quad(7) \\
& G_{\alpha, L, \alpha^{\prime}, L^{\prime}}^{R}\left(\boldsymbol{k}_{\|} ; t, t^{\prime}\right) \\
& \quad=\theta\left(t-t^{\prime}\right)\left[G_{\alpha, L, \alpha^{\prime}, L^{\prime}}^{>}\left(\boldsymbol{k}_{\|} ; t, t^{\prime}\right)-G_{\alpha, L, \alpha^{\prime}, L^{\prime}}^{<}\left(\boldsymbol{k}_{\|} ; t, t^{\prime}\right)\right],
\end{aligned}
$$

where $c_{L, \alpha^{j}, \boldsymbol{k}_{\|}}$is an electron annihilation operator which is the second-quantized form of the coefficient in Eq. (1) and $\theta\left(t-t^{\prime}\right)$ is a step function. We Fourier transform the Green's functions with respect to the time difference coordinate $\left(t-t^{\prime}\right)$. Then we can follow similar treatment of the Dyson equations $[7,8]$ and its extension to the MB space [9], except we have to use the correct modematching condition Eq. (5) at interfaces [10].

Finally we can calculate both the electron concentration and the current density at layer $L$ as follows,

$$
\begin{aligned}
n_{L}= & -\frac{2 i}{A \Delta} \sum_{\boldsymbol{k}_{\|}} \int \frac{d E}{2 \pi} \operatorname{Tr}\left[G_{L, L}^{<}\left(\boldsymbol{k}_{\|}, E\right)\right], \\
J_{L}= & \frac{2 e}{\hbar A} \sum_{\boldsymbol{k}_{\|}} \int \frac{d E}{2 \pi} 2 \operatorname{Re} \\
& \left\{\operatorname{Tr}\left[-t_{L, L+1} G_{L+1, L}^{<}, L\left(\boldsymbol{k}_{\|}, E\right)\right]\right\},
\end{aligned}
$$

where $e$ is the electronic charge, $\mathbf{T r}$ denotes trace of the matrix, and $t_{L, L+1}$ is a hopping matrix. Special care is necessary to evaluate Eq. (10), when the numbers of propagating (Bloch) modes from left to right and right to left are different.

To include space charge effect for selfconsistent calculation, the Poisson's equation is simultaneously solved.

\section{RESULTS AND DISCUSSION}

We focus on quantum transport in a $\mathrm{Si}$ and a GaAs $\mathrm{p}^{+}-\mathrm{n}^{+}$ITD as schematically illustrated in Figure 1. Impurity doping density in both electrodes is assumed $1.0 \times 10^{20} \mathrm{~cm}^{-3}$ and the temperature is $100 \mathrm{~K}$. No scattering effects are taken into account.

The calculated band structures of $\mathrm{GaAs}$ and $\mathrm{Si}$ bulk at $\boldsymbol{k}_{\|}=\mathbf{0}$ in the [ $\left[\begin{array}{lll}0 & 0 & 1\end{array}\right]$ direction are shown in Figures 2(a) and (b), respectively. For comparison, the band structures calculated by conventional two-band (2B) model are also shown by thin lines. Real $k_{z}$ is plotted in the right portion of the graph while imaginary wave vector in the left. It is found the $2 \mathrm{~B}$ model can comparatively well express the 


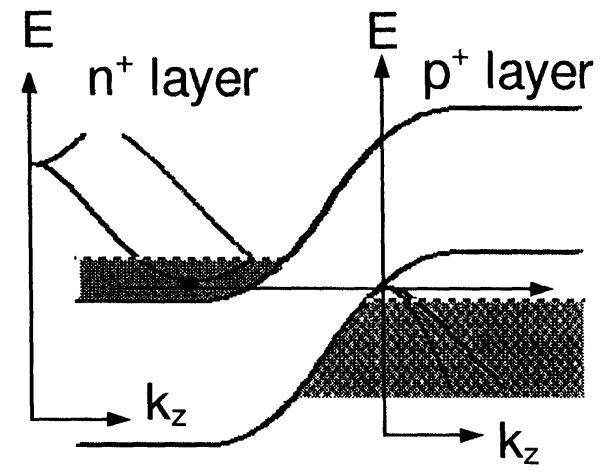

FIGURE 1 Schematic structure of a Si interband tunneling diode (ITD). Two propagating modes exist in the conduction band, whereas three modes exist in the valence band because the heavy hole band is doubly degenerated (excluding spin degeneracy).
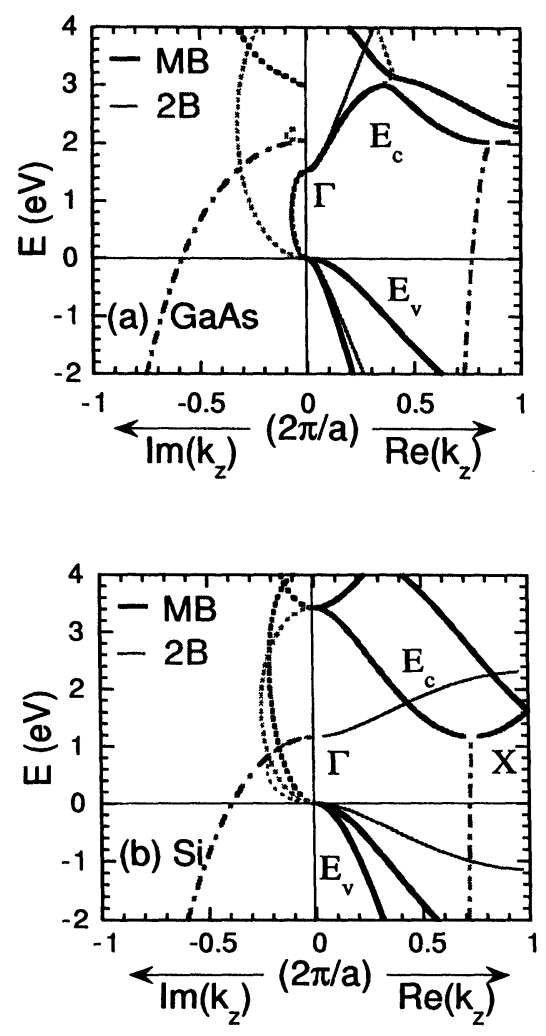

FIGURE 2 Complex band structures of (a) GaAS and (b) Si bulk along the [001] direction. Both the real bands (solid lines: $\operatorname{Im} k_{z}=0$ ) and the complex bands corresponding to evanescent modes (dashed lines: $\operatorname{Re} k_{z} \neq 0$ and $\operatorname{Im} k_{z} \neq 0$ ) are shown. The origin of the electron energy is set at the valence-band top of each matrial. band structure of GaAs in the vicinity of the band center ( $\Gamma$-point) compared with the MB model.

On the other hand, it is shown that $2 \mathrm{~B}$ model cannot express the indirect gap of $\mathrm{Si}$, while the $\mathrm{MB}$ model can reflect the realistic band structure. In $\mathrm{Si}$ bulk, for a given energy $E>1.1 \mathrm{eV}$, there are two electron modes, one of which has positive group velocity and the other negative, in the conduction band. In the valence band, the heavy hole $(\mathrm{HH})$ band is doubly degenerated since we have neglected the spin-orbit interaction. Not only the real bands, but also the complex modes should be considered at a tunneling interface to match electron waves existing in the $\mathrm{Si}$ crystal, since two electron modes exist in the conduction band, whereas three modes exist in the valence band.

Figure 3(a) shows comparison of $I-V$ characteristics of the GaAs ITD calculated by the MB model and the 2B model. Since the $2 B$ model well approximates the direct gap structure, there are small discrepancies between the two results. Tunneling mainly occurs near $\Gamma$-point where electrons in the conduction band with the $s$-orbital component propagates to the $p$-state in the valence band.

Figure 3(b) shows $I-V$ characteristics of the $\mathrm{Si}$ ITD calculated by the MB model, whereas Figure 3(c) shows those by the conventional 2B model. It should be emphasized that the peak current density of the MB model is much less than that of the $2 \mathrm{~B}$ model. The transmission state is shown in Figures 4(a) and (b) for the MB and 2B model, respectively, where $A_{N, 1}$ is an element of the spectral function which corresponds to the transmission probability. Since the $2 \mathrm{~B}$ model assumes the band extrema exist at the $\Gamma$ valley, electron in the $s$-state couples that in the $p_{z}$-state in the $\mathrm{p}^{+}$region. However, in an actual Si crystal, the contribution of the $s^{*}$ orbital to the $X$ conduction band minimum is fairly large. As shown in Figure 4(a), electrons in the $s^{*}, s$, and $p_{z}$ orbitals are affected with more decay which results in smaller tunneling probability. Consequently the MB model has smaller current density. The difference of the order of 5 in the peak 

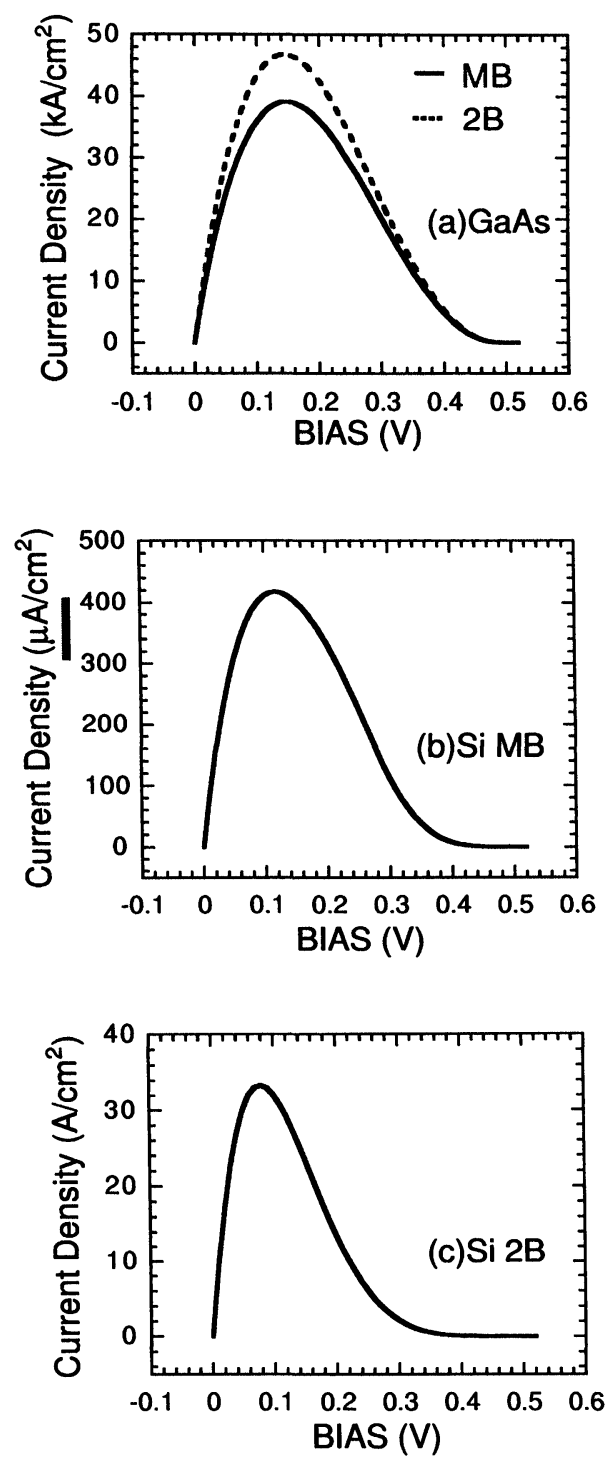

FIGURE 3 (a) Comparison of the $I-V$ characteristics of the GaAs ITD calculated by the MB model with the 2B model. (b) $I-V$ characteristics of the Si ITD calculated by the MB. The peak current density is considerably small. (c) $I-V$ characteristics of the Si ITD calculated by the 2B model.

current densities may be due to the TB parameterization [6], where the coupling of $s^{*}$ with neighboring $s$ states is neglected: $\left(s^{*} a \boldsymbol{R}|H| s^{*} c \boldsymbol{R}\right)=$ $\left(s^{*} a \boldsymbol{R}|H| s c \boldsymbol{R}\right)=\left(s a \boldsymbol{R}|H| s^{*} c \boldsymbol{R}\right)=0$.

Since the $\Gamma-\Gamma$ interband tunneling occurs in the case of GaAs ITDs, the difference of the interband tunneling mechanisms may be experimentally
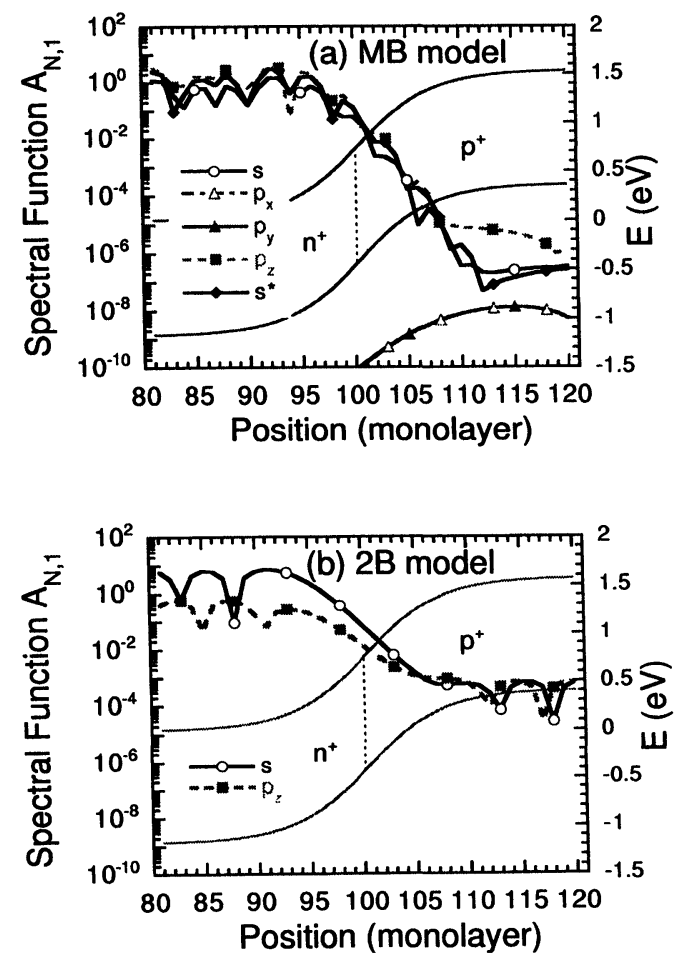

FIGURE 4 (a) Spectral function or transmission amplitude calculated by the multiband (MB) model. The $s^{*}$-component as well as the $s$ and $p_{z}$ are found to be dominant in the conduction band. (b) Spectral function by the $2 \mathrm{~B}$ model. Since both the conduction and the valence bands are assumed to be comprised of the $s$ and $p_{z}$-like atomic orbitals, the spectral function is larger than that in the MB model.

demonstrated by a comparison of the peak current density between the GaAs and Si ITD's with same structures.

\section{CONCLUSION}

We have studied multiband quantum transport in both GaAs and Si ITD's based on the nonequilibrium Green's functions, where full-band nature of realistic band structures and space charge effect are taken into account. It is found the conventional 2B model cannot analyze the interband tunneling from the $X$ band of $\mathrm{n}^{+}$Si to the $\Gamma$ band of $\mathrm{p}^{+}$(and vice versa), since the $2 \mathrm{~B}$ model cannot produce the indirect gap structure. On the other hand, the MB model can treat 
properly the interband tunneling process in the indirect gap material. It is also found that the transmissivity of the $X-\Gamma$ interband tunneling is considerably small, since it is mainly governed by the tunneling via $s^{*}$ and $p$ states different from the case of the $\Gamma-\Gamma$ interband tunneling where the tunneling through $s$ and $p$ states is dominant. The significant difference of the peak current densities between the $\mathrm{Si}$ and GaAs ITD's is predicted, which should be verified by further experimental efforts.

\section{References}

[1] Koga, J. and Toriumi, A. (1996). "Negative Differential Conductance in Three-Terminal Silicon Tunneling Device", Appl. Phys. Lett., 69, 1435-1437.

[2] Koga, J., Vanderstraeten, C., Takagi, S. and Toriumi, A. (1999). "New Approach to Negative Differential Conductance with High Peak-to-Valley Ratio in Silicon", Extended Abst. 1999 ICSSDM, Tokyo, pp. 350-351.
[3] Baba, T. and Uemura, T. (1997). "Surface Tunnel Transistors with Multiple Interband Tunneling Junctions", IEICE Trans. Electron., E80-C, 875-880.

[4] Heine, V. (1960). "On the General Theory of Surface States and Scattering of Electrons in Solids", Proc. Phys. Soc., 81, 300-310.

[5] Ogawa, M., Sugano, T. and Miyoshi, T. (1998). "Multiband Quantum Transport with $\Gamma-X$ ValleyMixing via Evanescent States", Solid St. Electron., 42, $1527-1532$.

[6] Vogl, P., Hjalmarson, H. P. and Dow, H. D. (1983). “A Semi-Empirical Tight-Binding Theory of the Electronic Structure of Semiconductors", J. Phys. Chem. Solids, 44, $365-378$.

[7] Caroli, C., Combescot, R., Nozieres, P. and Saint-James, D. (1971). "Direct Calculation of the Tunneling Current", J. Phys. C: Solid St. Phys., 4, 916-929.

[8] Sols, F., Macucci, M., Ravaioli, U. and Hess, K. (1989). "Theory for a Quantum Modulated Transistor", J. Appl. Phys., 66, 3892-3906.

[9] Lake, R., Klimeck, G., Bowen, R. C. and Jovanovic, D. (1997). "Single and Multiband Modeling of Quantum Electron Transport Through Layered Semiconductor Devices", J. Appl. Phys., 81, 7845-7869.

[10] Ogawa, M., Sugano, T. and Miyoshi, T. (1999). "Multiband Modeling of Quantum Electron Transport Based on the Green Function Theory", Inst. Phys. Conf. Ser., 162, Ed. Sakaki, H., 319-324. 

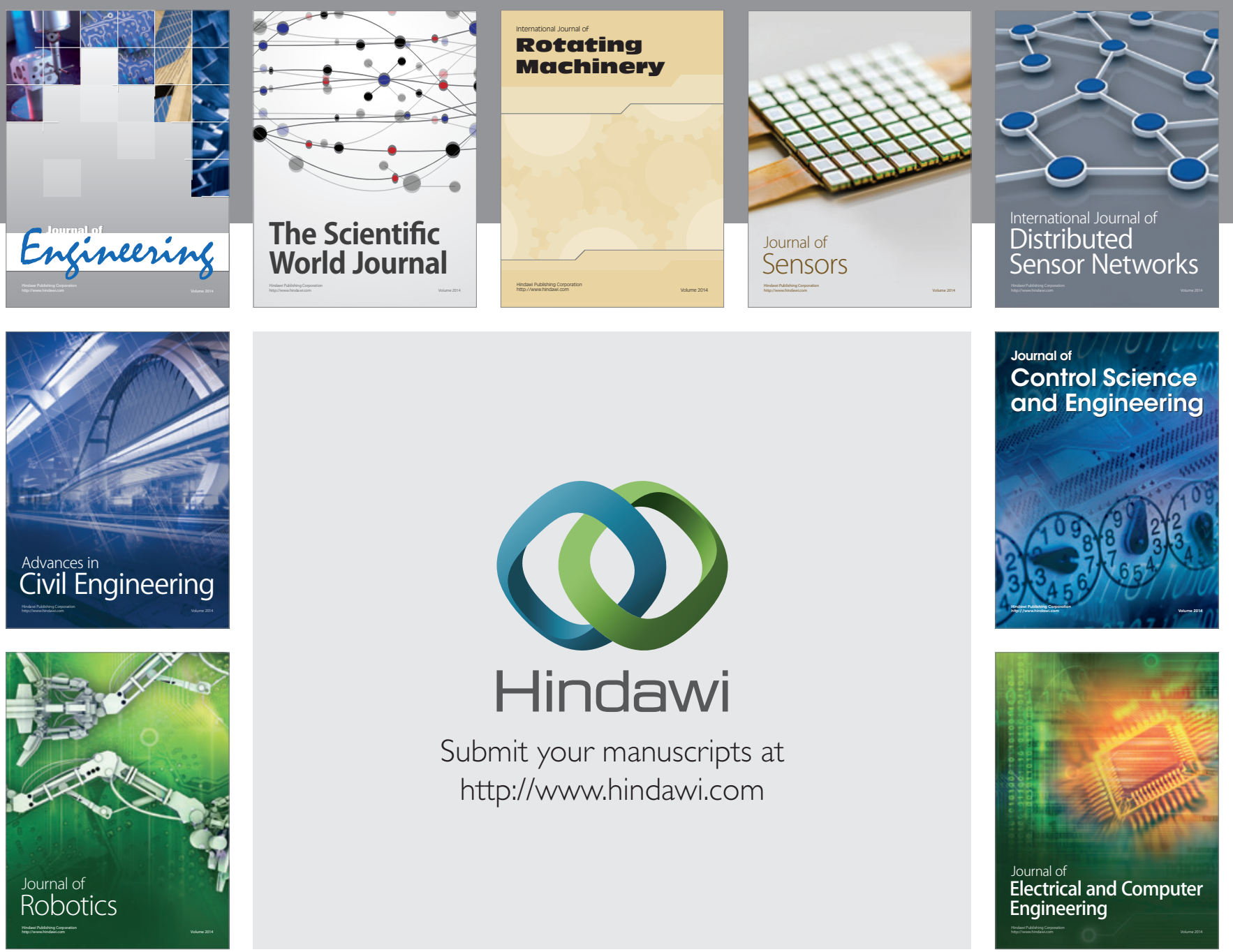

Submit your manuscripts at

http://www.hindawi.com
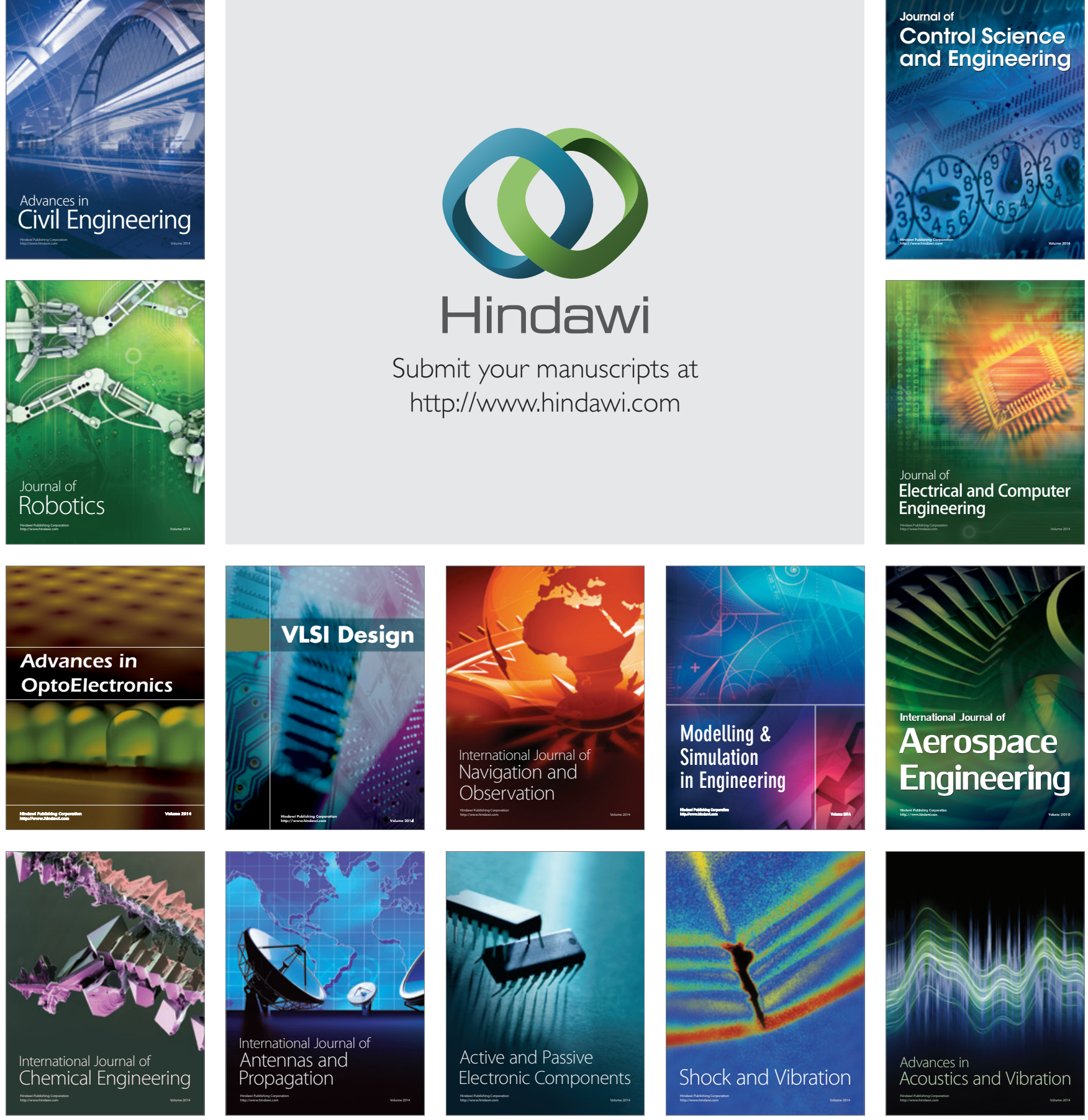